\title{
Specification of Fractional Poincare' Inequalities for the Sequence Measures Generalization
}

\author{
Abdelilah Kamal H. Sedeeg ${ }^{1,2}$, Shawgy H. Abd Alla ${ }^{3}$ \\ ${ }^{1}$ Mathematics Department, Faculty of Education, Holy Quran and Islamic Sciences University, Khartoum, Sudan \\ ${ }^{2}$ Mathematics Department, Faculty of Sciences and Arts-Almikwah, Albaha University, Albaha, Saudi Arabia \\ ${ }^{3}$ Mathematics Department, Faculty of Sciences, Sudan University of Science and Technology, Khartoum, Sudan
}

Email address:

aelilah63@hotmail.com (A. K. H. Sedeeg), shawgy2020@gmail.com (S. H. A. Alla)

To cite this article:

Abdelilah Kamal H. Sedeeg, Shawgy H. Abd Alla. Specification of Fractional Poincare' Inequalities for the Sequence Measures Generalization. American Journal of Applied Mathematics. Vol. 5, No. 3, 2017, pp. 57-67. doi: 10.11648/j.ajam.20170503.11

Received: October 13, 2016; Accepted: November 8, 2016; Published: June 12, 2017

\begin{abstract}
The Fractional Poincare' inequalities in $\mathbb{R}^{n}$ are endowed with a fairly general sequence measure. We show a control of $L^{2}$ norm by a non-Local quantity. The assumption on the sequence measure is that it satisfies the classical Poincare' inequality, with general results. We also verify quantity of the tightness at infinity provided by the control on the fractional derivative in terms of a sequence of a weight growing at infinity. The illustration goes to the generator of the OrnsteinUhlenbeck semi group and some estimates of its powers.
\end{abstract}

Keywords: Poincare Inequalities, Non-Local Inequalities, Fractional Powers, Sequence Measure

\section{Introduction}

Fractional diffusions naturally appear in many models (see [1-7]). The theory also appears naturally in mathematics: in probability they appear in the important class of infinitely divisible Markov processes (see [8]); in analysis they naturally appear in the study of singular integral operators (see[3-6]) as well as in the so-called "Dirichlet-to-Neuman" boundary value problem and in [9] (see also [10, 11]). Clément Mouhot, Emmanuel Russ and Yannick Sire [12] prove a Poincaré inequality on $\mathbb{R}^{\mathrm{n}}$, endowed with a measure $\mathrm{M}(\mathrm{x}) \mathrm{dx}$, involving non-local quantities on the right-hand side in the spirit of Gagliardo semi-norms for Sobolev spaces $\mathrm{W}^{\mathrm{s}, \mathrm{p}}\left(\mathbb{R}^{\mathrm{n}}\right)$ with fractional order $\mathrm{s} \in(0,1)$ (see [13]). Poincaré inequality for the non-local fractional energy associated with such fractional diffusion is, therefore, a natural and interesting question since this inequality governs the spectral gap of the underlying operator and the speed of (fractional) diffusion towards an equilibrium. The work of [12] is verified, using the same strong methodology, applying the sequence measure of a $C^{2}$-function.

$M$ denotes a positive weight in $L^{1}\left(\mathbb{R}^{n}\right)$. By $L^{2}\left(\mathbb{R}^{n}, M\right)$, the space of measurable functions on $\mathbb{R}^{\mathrm{n}}$ is signified which are square integrable with respect to the sequence measure $\mathrm{M}\left(\mathrm{x}_{n}\right) \mathrm{dx}_{n}$, by $\mathrm{L}_{0}^{2}\left(\mathbb{R}^{\mathrm{n}}, \mathrm{M}\right)$ the subspace of functions of
$\mathrm{L}^{2}\left(\mathbb{R}^{\mathrm{n}}, \mathrm{M}\right)$ such that $\int_{\mathbb{R}^{\mathrm{n}}} \mathrm{f}\left(\mathrm{x}_{n}\right) \mathrm{M}\left(\mathrm{x}_{n}\right) \mathrm{dx}_{n}=0$, and by $H^{1}\left(\mathbb{R}^{n}, M\right)$, the Sobolev space of functions in $L^{2}\left(\mathbb{R}^{n}, M\right)$, the weak derivative of which belongs to $L^{2}\left(\mathbb{R}^{2}, M\right)$. Finally, for any measurable subset $A \subset \mathbb{R}^{n}$ by $L^{2}(A, M)$ the obvious restriction of the definition above to the set $\mathrm{A}$ is designated.

The assumption is that $M$ is a sequence $\mathrm{C}^{2}$ function and that this sequence measure $\mathrm{M}$ satisfies the usual Poincaré inequality: there exists a constant $\lambda(\mathrm{M})>0$ such that $\forall f \in H^{1}\left(\mathbb{R}^{n}, M\right)$,

$$
\begin{gathered}
\int_{\mathbb{R}^{n}}\left|\nabla f\left(x_{n+1}\right)\right|^{2} M\left(x_{n+1}\right) d x_{n+1} \geq \lambda(M) \int_{\mathbb{R}^{n}} \mid f\left(x_{n+1}\right)- \\
\left.\int_{\mathbb{R}^{n}} f\left(x_{n}\right) M\left(x_{n}\right) d x_{n}\right|^{2} M\left(x_{n+1}\right) d x_{n+1}
\end{gathered}
$$

If the sequence measure $M$ can be written $M=e^{-V}$, this inequality is known to hold (see [14-29]), whenever there exists $\epsilon>0, c>0$ and $R>0$ so that.

$$
\forall\left|x_{n}\right| \geq R,(1-\epsilon)\left|\nabla V\left(x_{n}\right)\right|^{2}-\Delta V \geq c
$$

The inequality (1) holds(see [12]), for instance, when $M=$ $(2 \pi)^{-n / 2} \exp \left(-\left|x_{n}\right|^{2} / 2\right)$ is the Gaussian measure, but also when $M\left(x_{n}\right)=e^{-\left|x_{n}\right|}$, and more generally when

$$
M\left(x_{n}\right)=e^{-\left|x_{n}\right|^{1+\epsilon}} \text { with } \epsilon>0 \text {. If } V \text { is convex, }|V|<\infty \text {, then }
$$

$$
\operatorname{Hess}(\mathrm{V}) \geq \operatorname{cstId}
$$


and the sequence measure $\mathrm{M}\left(x_{n}\right) \mathrm{d} x_{n}$ satisfies the logSobolev inequality, which in turn implies (1) (see [22], [12]).

The Poincaré inequality (1) admits the following selfimprovement for completeness

Proposition (1.1): Assume that there exists $\varepsilon>0$ such that

$$
\frac{(1-\varepsilon)|\nabla V|^{2}}{2}-\nabla V \overline{x_{n} \rightarrow \infty}+\infty, \quad M=e^{-V}
$$

Then there exists $\lambda^{\prime}(M)>0$ such that, for all functions $f \in$ $L_{0}^{2}\left(\mathbb{R}^{n}, M\right) \cap H^{1}\left(\mathbb{R}^{n}, M\right)$ :

$$
\begin{gathered}
\iint_{\mathbb{R}^{n}}\left|\nabla f\left(x_{n}\right)\right|^{2} M\left(x_{n}\right) d x_{n} \geq \lambda^{\prime}(M) \int_{\mathbb{R}^{n}}\left|f\left(x_{n}\right)\right|^{2}(1+ \\
\left.\left|\nabla \operatorname{In} M\left(x_{n}\right)\right|^{2}\right) M\left(x_{n}\right) d x_{n}
\end{gathered}
$$

The Authors in [12] generalize the inequality (1) in the strengthened form of Proposition (1.1), replacing the $\mathrm{H}^{1}$ semi-norm, in the left-hand side, by a non-local expression in the flavour of the Gagliardo semi-norms.

The following theorem are shown below (see [12]).

Theorem (1.2): Assumes that $\mathrm{M}=\mathrm{e}^{-\mathrm{V}}$ is a $\mathrm{C}^{2}$ positive $\mathrm{L}^{1}$ function which satisfies (3). Let $\epsilon>0$. Then there exist $\lambda_{2-\epsilon}(\mathrm{M})>0$ and $\delta(\mathrm{M})$ (constructive from our proof and the usual Poincaré constant $\lambda(\mathrm{M})$ such that, for any function $\mathrm{f}$ belonging to a dense subspace of $\mathrm{L}_{0}^{2}\left(\mathbb{R}^{\mathrm{n}}, \mathrm{M}\right)$, the formula:

$$
\begin{aligned}
& \iint_{\mathbb{R}^{n} \times \mathbb{R}^{n}} \frac{\left|f\left(x_{n}\right)-f\left(x_{n+1}\right)\right|^{2}}{\left|x_{n}-x_{n+1}\right|^{n+2-\epsilon}} M\left(x_{n}\right) e^{-\delta(M)\left|x_{n}-x_{n+1}\right|} d x_{n} d x_{n+1} \\
& \geq \lambda_{2-\epsilon}(M) \int_{\mathbb{R}^{n}}\left|f\left(x_{n}\right)\right|^{2}\left(1+\left|\nabla \operatorname{In} M\left(x_{n}\right)\right|^{2-\epsilon}\right) M\left(x_{n}\right) d x_{n}
\end{aligned}
$$

In particular (5) is satisfied for any function $f$ with zero average (see [12]) belonging to the domain of the operator $L=-\Delta-\nabla V . \nabla$. Functions of this domain with zero integral with respect to $M\left(x_{n}\right) d x_{n}$ are dense in $L_{0}^{2}\left(\mathbb{R}^{n}, M\right)$.

Observe that the right-hand side of (5) involves a fractional moment of order $(1+\epsilon)$ related to the homogeneity of the semi-norm appearing in the left-hand side. One could expect (see [12]) in the left-hand side of (5) the Gagliardo semi-norm for the fractional Sobolev space $H^{(2-\epsilon) / 2}\left(\mathbb{R}^{n}, M\right)$, namely

$$
\iint_{\mathbb{R}^{n} \times \mathbb{R}^{n}} \frac{\left|f\left(x_{n}\right)-f\left(x_{n+1}\right)\right|^{2}}{\left|x_{n}-x_{n+1}\right|^{n+2-\epsilon}} M\left(x_{n}\right) M\left(x_{n+1}\right) d x_{n} d x_{n+1}
$$

Notice that, instead of this semi-norm, (see [12]) obtain a "non-symmetric" expression. However, our norm is more natural: one should think of the sequence measure over $x_{n+1}$ as the Lévy measure, and thesequence measure over $x_{n}$ as the ambient measure. It is thus emphasized that the sequence measure is rather general (see [12]) and in particular, as a corollary of Theorem (1.2), an automatic improvement of the Poincare inequality (1) is obtained by:

$$
\begin{gathered}
\iint_{\mathbb{R}^{n} \times \mathbb{R}^{n}} \frac{\left|f\left(x_{n}\right)-f\left(x_{n+1}\right)\right|^{2}}{\left|x_{n}-x_{n+1}\right|^{n+2-\epsilon}} M\left(x_{n}\right) M\left(x_{n+1}\right) d x_{n} d x_{n+1} \\
\geq \lambda_{1+\epsilon}(M) \int_{\mathbb{R}^{n}}\left|f\left(x_{n}\right)\right|^{2} M\left(x_{n}\right) d x_{n} .
\end{gathered}
$$

The question of obtaining Poincaré-type inequalities (or more generally entropy inequalities) for Lévy operators was studied in the probability community in the last decades. For instance it was proved by $\mathrm{Wu}$ [23] and Chafaï [24] that

$$
\begin{aligned}
& \operatorname{Ent}_{\mu}^{\Phi}(f) \leq \int \Phi^{n}(f) \nabla f \cdot \sigma \cdot \nabla f d \mu \\
& \quad+\iint D_{\Phi}\left(f\left(x_{n}\right), f\left(x_{n}\right.\right. \\
& \left.\left.\quad+z_{n}\right)\right) d v_{\mu}\left(z_{n}\right) d v_{\mu}\left(x_{n}\right)
\end{aligned}
$$

(see also [25]) with

$$
\operatorname{Ent}_{\mu}^{\Phi}(f)=\int \Phi(f) d \mu-\Phi\left(\int f d \mu\right)
$$

And $D_{\Phi}$ is the so-called Bregman distance associated to $\Phi$ :

$$
D_{\Phi}(a, a-\epsilon)=\Phi(a)-\Phi(a-\epsilon)-\Phi^{\prime}(a-\epsilon)(\epsilon),
$$

Where $\Phi$ is some well-suited functional with convexity properties, $\sigma$ the matrix of diffusion of the process, $\mu$ a rather general measure, and $v_{\mu}$ the (singular) Lévy measure associated to $\mu$. Choosing $\Phi\left(x_{n}\right)=x_{n}^{2}$ and $\sigma=0$ yields a Poincaré inequality for this choice of measure $\mu, v_{\mu}$. The improvement of this approach is that it does not impose any link between sequence measure $M$ on $x_{n}$ and the singular measure $\left|z_{n}\right|^{-n-(2-\epsilon)}$ on $z_{n}=x_{n}-x_{n+1}$. (see [12]).

Remark (1.3): Note that the exponentially decaying factor $e^{-\delta(M)\left|x_{n}-x_{n+1}\right|}$ in (5) also improvesthe inequality as compared to what is expected from Poincaré inequality for Lévy measures (see [12]). Other extensions in progress are to allow more general singularities than the Martin Riesz kernel $\frac{1}{\left|x_{n}-x_{n+1}\right|^{n+2-\epsilon}}$ (see [26]) and to develop an $L^{P}$ theory of the previous inequalities.

The proof of [12] heavily relies on fractional powers of a (suitable generalization of the) Ornstein-Uhlenbeck operator, which is defined by:

$$
L f=-M^{-1} \operatorname{div}(M \nabla f)=-\nabla f-\nabla \ln M \cdot \nabla f,
$$

for all $f \in D\left(L^{*}\right):=\left\{\mathrm{g} \in H^{1}\left(\mathbb{R}^{n}, M\right) ;(1 / \sqrt{M}) \operatorname{div}(M \nabla \mathrm{g}) \in\right.$ $\left.L^{2}\left(\mathbb{R}^{n}\right)\right\}$. One therefore has, for all $f \in \mathcal{D}\left(L^{*}\right)$ and $g \in$ $H^{1}\left(\mathbb{R}^{n}, M\right)$,

$$
\begin{aligned}
\int_{\mathbb{R}^{n}} L f\left(x_{n}\right) g\left(x_{n}\right) M\left(x_{n}\right) d x_{n} \\
=\int_{\mathbb{R}^{n}} \nabla f\left(x_{n}\right) \cdot \nabla g\left(x_{n}\right) M\left(x_{n}\right) d x_{n} .
\end{aligned}
$$

It is obvious that $L$ is symmetric and nonnegative on $L^{2}\left(\mathbb{R}^{n}, M\right)$, which allows to define the usual power $L^{\beta}$ for any $\beta \in(0,1)$ by means of spectral theory. Note that $L^{(1+\epsilon) / 2}$ is not the symmetric operator associated to the Dirichlet form

$$
\iint_{\mathbb{R}^{n} \times \mathbb{R}^{n}} \frac{\left|f\left(x_{n}\right)-f\left(x_{n+1}\right)\right|^{2}}{\left|x_{n}-x_{n+1}\right|^{n+2-\epsilon}} M\left(x_{n}\right) d x_{n} d x_{n+1} .
$$

The Authors in [12] proved Theorem (1.2) by first establishing $L^{2}$ off-diagonal estimates of Gaffney type on the 
resolvent of $L$ on $L^{2}\left(\mathbb{R}^{n}, M\right)$. These estimates are needed, since Gaussian pointwise estimates on the kernel of the operator $L$ are not available.Then, they bound the quantity,

$$
\int_{\mathbb{R}^{n}}\left|f\left(x_{n}\right)\right|^{2}\left(1+\left|\nabla \ln M\left(x_{n}\right)\right|^{2-\epsilon}\right) M\left(x_{n}\right) d x_{n},
$$

In terms of $\left\|L^{*(2-\epsilon) / 4} f\right\|_{L^{2}\left(\mathbb{R}^{n}, M\right)}^{2}$. This will be obtained by an abstract argument of functional calculus based on rewriting in a suitable way the conclusion of Proposition (1.1). Finally, using the $L^{2}$ off-diagonal estimates for the kernel of $L$, it is establish that

$$
\leq C \iiint_{\mathbb{R}^{n} \times \mathbb{R}^{n}} \frac{\left|f\left(x_{n}\right)-f\left(x_{n+1}\right)\right|^{2}}{\left|x_{n}-x_{n+1}\right|^{n+2-\epsilon}} M\left(x_{n}\right) d x_{n} d x_{n+1},
$$

Which concludes the proof. They also borrow methods from harmonic analysis. This seems not so common in the field of Poincaré and log-Sobolev inequalities, where standard techniques rely on global functional inequalities, see for instance the powerful so-called $\Gamma_{2}$-calculus of Bakry and Émery [27].

\section{Resolvent of $L^{*}$ by the Off-Diagonal $L^{2}$ Estimates}

Recall that for every $f \in \mathcal{D}\left(L^{*}\right)$, it is defined

$$
L^{*} f=-M^{-1} \operatorname{div}(M \nabla f)=-\Delta f-\nabla \ln M \cdot \nabla f
$$

From the fact that $L^{*}$ is self-adjointand nonnegative on $\mathrm{L}^{2}\left(\mathbb{R}^{\mathrm{n}}, \mathrm{M}\right)$ we have:

$$
\left\|\left(L^{*}-\mu\right)^{-1}\right\|_{L^{2}\left(\mathbb{R}^{\mathrm{n}}, \mathrm{M}\right)} \leq \frac{1}{\operatorname{dist}\left(\mu, \sum\left(L^{*}\right)\right)}
$$

Where $\Sigma\left(L^{*}\right)$ denotes the spectrum of $L$, and $\mu \notin \Sigma(L)$. Then it can be deduced that $\left(I+\left(t^{2}-1\right) L^{*}\right)^{-1}$ is bounded with norm less than 1 for all $t^{2}>1$. Since $\left(t^{2}-1\right) L^{*}(\mathrm{I}+$ $\left.\left(t^{2}-1\right) L^{*}\right)^{-1}=\mathrm{I}-\left(\mathrm{I}+\left(t^{2}-1\right) L^{*}\right)^{-1}$, the same is true for $\left(t^{2}-1\right) L^{*}\left(\mathrm{I}+\left(t^{2}-1\right) L^{*}\right)^{-1}=\mathrm{I}-\left(I+\left(t^{2}-1\right) L^{*}\right)^{-1}$ with a norm less than 2 . Moreover, $\left(I+\left(t^{2}-1\right) L^{*}\right)^{-1} f \in$ $H^{1}\left(\mathbb{R}^{n}, M\right)$.

Actually, when $f \in L^{2}\left(\mathbb{R}^{n}, M\right)$ is supported in a closed set $E \subset \mathbb{R}^{n}$ and $F \subset \mathbb{R}^{n}$ is a closed subset disjoint from $E$, a much more precise estimate on the $L^{2}$ norm of (I+ $\left.\left(t^{2}-1\right) L\right)^{-1} f$ and $\left(t^{2}-1\right) L^{*}\left(I+\left(t^{2}-1\right) L^{*}\right)^{-1} f$ on $F$ can be given. Here are these $L^{2}$ off-diagonal estimates for the resolventof $L^{*}$.

Lemma (2.1): There exists $C_{1}=C_{1}(M)>0$ with the following property: for all compact disjoint subsets $E, F \subset$ $\mathbb{R}^{n}, F$ bounded, with $\operatorname{dist}(E, F)=: t+\epsilon, \epsilon>0$, all functions $f \in L^{2}\left(\mathbb{R}^{n}, M\right)$ supported in $E$ and all $t^{2}>1$

$$
\begin{aligned}
& \left\|\left(\mathrm{I}+\left(t^{2}-1\right) L^{*}\right)^{-1} f\right\|_{L^{2}(F, M)} \\
& \quad+\left\|\left(t^{2}-1\right) L^{*}\left(\mathrm{I}+\left(t^{2}-1\right) L^{*}\right)^{-1} f\right\|_{L^{2}(F, M)} \\
& \quad \leq 8 e^{-C_{1} \frac{t+\epsilon}{\sqrt{t}}}\|f\|_{L^{2}(E, M)}
\end{aligned}
$$

Note that, in different contexts, this kind of estimate, originating in [28], turns out to be a powerful tool, especially when no pointwise upper estimate on the kernel of the semigroup generated by $L^{*}$ is available (see [29-31]). Since no reference for these off-diagonal estimates for the resolvent of $L^{*}$ could be obtained, here a general proof [12] is provided.

Proof of Lemma (2.1): As in [32] it is argued, since $\left(\mathrm{I}+\left(t^{2}-1\right) L^{*}\right)^{-1}$ is bounded with norm less than 1 for all $\mathrm{t}^{2}>1$ it is clearly enough to restrictto $\epsilon>0$.

Defineu $\left(t^{2}-1\right)=\left(I+\left(t^{2}-1\right) L^{*}\right)^{-1} f$, so that, for all functions $v \in H^{1}\left(\mathbb{R}^{n}, M\right)$,

$$
\begin{aligned}
& \int_{\mathbb{R}^{n}} u_{t^{2}-1}\left(x_{n}\right) v\left(x_{n}\right) M\left(x_{n}\right) d x_{n} \\
& +\left(t^{2}-1\right) \int_{\mathbb{R}^{n}} \nabla u_{t^{2}-1}\left(x_{n}\right) \cdot \nabla v\left(x_{n}\right) M\left(x_{n}\right) \\
& \quad=\int_{\mathbb{R}^{n}} f\left(x_{n}\right) v\left(x_{n}\right) M\left(x_{n}\right) d x_{n}
\end{aligned}
$$

Fix now a nonnegative function $\eta \in \mathcal{D}\left(\mathbb{R}^{n}\right)$ vanishing on $E$. Since $f$ is supported in $E$, applying (7) with $v=$ $\eta^{2} u_{t^{2}-1}\left(\right.$ remember that $\left.u_{t^{2}-1} \in H^{1}\left(\mathbb{R}^{n}, M\right)\right)$ yields,

$$
\begin{gathered}
\int_{\mathbb{R}^{n}} \eta^{2}\left(x_{n}\right)\left|u_{t^{2}-1}\left(x_{n}\right)\right|^{2} M\left(x_{n}\right) d x_{n} \\
+\left(t^{2}-1\right) \int_{\mathbb{R}^{n}} \nabla u_{t^{2}-1}\left(x_{n}\right) \\
\cdot \nabla\left(\eta^{2} u_{t^{2}-1}\right) M\left(x_{n}\right) d x_{n}=0
\end{gathered}
$$

which implies:

$$
\begin{gathered}
\int_{\mathbb{R}^{n}} \eta^{2}\left(x_{n}\right)\left|u_{t^{2}-1}\left(x_{n}\right)\right|^{2} M\left(x_{n}\right) d x_{n} \\
+\left(t^{2}-1\right) \int_{\mathbb{R}^{n}} \eta^{2}\left(x_{n}\right)\left|\nabla u_{t^{2}-1}\left(x_{n}\right)\right|^{2} M\left(x_{n}\right) d x_{n} \\
=-2\left(t^{2}-1\right) \int_{\mathbb{R}^{n}} \eta\left(x_{n}\right) u_{t^{2}-1}\left(x_{n}\right) \nabla \eta\left(x_{n}\right) \cdot \nabla u_{t^{2}-1}\left(x_{n}\right) M\left(x_{n}\right) d x_{n} \\
\leq\left(t^{2}-1\right) \int_{\mathbb{R}^{n}}\left|u_{t^{2}-1}\left(x_{n}\right)\right|^{2}\left|\nabla \eta\left(x_{n}\right)\right|^{2} M\left(x_{n}\right) d x_{n} \\
+\left(t^{2}\right. \\
-1) \int_{\mathbb{R}^{n}} \eta^{2}\left(x_{n}\right)\left|\nabla u_{t}\left(x_{n}\right)\right|^{2} M\left(x_{n}\right) d x_{n}
\end{gathered}
$$

hence 


$$
\left(t^{2}-1\right) \int_{\mathbb{R}^{n}}\left|u_{t^{2}-1}\left(x_{n}\right)\right|^{2}\left|\nabla \eta\left(x_{n}\right)\right|^{2} M\left(x_{n}\right) d x_{n}
$$

Let $\xi$ be such that $\xi=0$ on $E$ and $\xi$ nonnegative so that

$$
\int_{\mathbb{R}^{\mathrm{n}}}\left|\mathrm{e}^{(2-\epsilon) \xi}-1\right|^{2}\left|\mathrm{u}_{t^{2}-1}\left(x_{n}\right)\right|^{2} \mathrm{M}\left(x_{n}\right) \mathrm{d} x_{n} \leq(2-\epsilon)^{2}\left(t^{2}-1\right) \int_{\mathbb{R}^{\mathrm{n}}}\left|\mathrm{u}_{t^{2}-1}\left(x_{n}\right)\right|^{2}\left|\nabla \xi\left(x_{n}\right)\right|^{2} \mathrm{e}^{2(2-\epsilon) \xi\left(x_{n}\right)} \mathrm{M}\left(x_{n}\right) \mathrm{d} x_{n} .
$$

Taking $\epsilon=2-1 /\left(2 \sqrt{t^{2}-1}\|\nabla \xi\|_{\infty}\right)$, one obtains:

$$
\int_{\mathbb{R}^{\mathrm{n}}}\left|\mathrm{e}^{(2-\epsilon) \xi\left(x_{n}\right)}-1\right|^{2}\left|\mathrm{u}_{t^{2}-1}\left(x_{n}\right)\right|^{2} \mathrm{M}\left(x_{n}\right) \mathrm{d} x_{n} \leq \frac{1}{4} \int_{\mathbb{R}^{n}}\left|u_{t^{2}-1}\left(x_{n}\right)\right|^{2} e^{2(2-\epsilon) \xi\left(x_{n}\right)} M\left(x_{n}\right) d x_{n} .
$$

Using the fact that the norm of $\left(I+\left(t^{2}-1\right) L^{*}\right)^{-1}$ is bounded by 1 uniformly int $t^{2}>1$, this gives:

$$
\begin{gathered}
\left\|e^{(2-\epsilon) \xi} u_{t^{2}-1}\right\|_{L^{2}\left(\mathbb{R}^{2}, M\right)} \leq\left\|\left(e^{(2-\epsilon) \xi}-1\right) u_{t^{2}-1}\right\|_{L^{2}\left(\mathbb{R}^{n}, M\right)}+\left\|u_{t^{2}-1}\right\|_{L^{2}\left(\mathbb{R}^{n}, M\right)} \\
\leq \frac{1}{2}\left\|e^{(2-\epsilon) \xi} u_{t^{2}-1}\right\|_{L^{2}\left(\mathbb{R}^{n}, M\right)}+\|f\|_{L^{2}\left(\mathbb{R}^{n}, M\right)}
\end{gathered}
$$

therefore

$$
\int_{\mathbb{R}^{n}}\left|e^{(2-\epsilon) \xi\left(x_{n}\right)}\right|^{2}\left|u_{t^{2}-1}\left(x_{n}\right)\right|^{2} M\left(x_{n}\right) d x_{n} \leq 4 \int_{\mathbb{R}^{n}}\left|f\left(x_{n}\right)\right|^{2} M\left(x_{n}\right) d x_{n}
$$

Suppose now $\xi$ such that $\xi=0$ on $E$ as before and additionally that $\xi=1$ on $F$ ( $\eta$ is then compactly supported from the fact that $F$ is bounded). It can trivially be chosen with $\|\nabla \xi\|_{\infty} \leq C /\left(\left(t^{2}-1\right)+\epsilon\right)$, which yields the desired conclusion for the $L^{2}$ norm of $\left(I+\left(t^{2}-1\right) L^{*}\right)^{-1} f$ with a factor 4 in the right-hand side. Since $\left(t^{2}-1\right) L^{*}(I+$ $\left.\left(t^{2}-1\right) L^{*}\right)^{-1} f=f-\left(I+\left(t^{2}-1\right) L^{*}\right)^{-1} f$, the desired inequality with a factor 8 readily follows.

Remark (2.2): Arguing similarly, we could also obtain analogous gradient estimates for $\| \sqrt{t^{2}-1} \nabla(\mathrm{I}+$ $\left.\left(t^{2}-1\right) \boldsymbol{L}^{*}\right)^{-1} f \|_{L^{2}(F, M)}$.

\section{Control of $\left\|L^{*(2-\epsilon) / 4} f\right\|_{L^{2}\left(\mathbb{R}^{n}, M\right)}$ of}

\section{Fractional Powers of $L^{*}$}

This section is devoted to the control of the $L^{2}$ norm of fractional powers of $L^{*}$. This is the cornerstone of the proof of Theorem (1.2). In the functional calculus theory of sectorial operators $L^{*}$, fractional powers (see [12]) are defined as follows (see [12]):

$$
\begin{array}{r}
\forall \epsilon_{1}>0, \quad L^{* 1-\epsilon_{1}} f= \\
\frac{1}{\Gamma\left(\epsilon_{1}\right)} \int_{0}^{\infty}\left(t^{2}-1\right)^{-\left(1-\epsilon_{1}\right)} L^{*} e^{-L^{*}\left(t^{2}-1\right)} f d\left(t^{2}-1 \mid\right)
\end{array}
$$

They can also be defined in terms of the resolvent by the Balakrishnan formulation (see instance [12]):

$$
\forall \epsilon_{1}>0, \quad L^{* 1-\epsilon_{1}} f=\frac{\sin \left(\pi \epsilon_{1}\right)}{\pi} \int_{0}^{\infty} \lambda^{\epsilon_{1}} L^{*}\left(L^{*}+\lambda \mathrm{I}\right)^{-1} f d \lambda
$$

As the representations (9) or (10) are redundant; instead reliance shall be on the powerful tool of the so-called "quadratic estimates" obtained in the functional calculus (see [12]).This is the object of the general next lemma.
Lemma (3.1): Let $\in>0$. There exists $\tilde{C}_{3}=\tilde{C}_{3}(M)>0$ such that, for all $f \in \mathcal{D}\left(L^{*}\right)$,

$$
\begin{aligned}
& \left\|L^{*(2-\epsilon) / 4} f\right\|_{L^{2}\left(\mathbb{R}^{n}, M\right)}^{2} \leq C_{3} \int_{0}^{+\infty}\left(t^{2}-1\right)^{-\frac{\epsilon-4}{2}} \|\left(t^{2}-\right. \\
& \text { 1) } L^{*}\left(I+\left(t^{2}-1\right) L^{*}\right)^{-1} f \|_{L^{2}\left(\mathbb{R}^{n}, M\right)}^{2} d\left(t^{2}-1\right)
\end{aligned}
$$

Proof: Let $\mu \in\left(0, \frac{\pi}{2}\right)$, and

$$
\sum_{\mu^{+}}=\left\{z_{n} \in \mathbb{C}^{*} ;\left|\arg z_{n}\right|<\mu\right\} .
$$

Let $\psi$ be a holomorphic function in $H^{\infty}\left(\sum_{\mu^{+}}\right)$such that for some $C, \sigma, \tau>0$,

$$
\left|\psi\left(z_{n}\right)\right| \leq C \inf \left\{\left|z_{n}\right|^{\sigma},\left|z_{n}\right|^{-\tau}\right\},
$$

for any $z_{n} \in \Sigma_{\mu^{+}}$. Since $L^{*}$ is positive self-adjoint operator on $L^{2}\left(\mathbb{R}^{n}, M\right)$ and $L^{*}$ is one-to-one on $L_{0}^{2}\left(\mathbb{R}^{n}, M\right)$ by (1), one has by the spectral theorem,

$$
\|F\|_{L^{2}\left(\mathbb{R}^{n}, M\right)}^{2} \leq C \int_{0}^{+\infty}\left\|\psi\left(\left(t^{2}-1\right) L^{*}\right) F\right\|_{L^{2}\left(\mathbb{R}^{n}, M\right)}^{2} \frac{d}{d\left(t^{2}-1\right)}
$$

Whenever $F \in L_{0}^{2}\left(\mathbb{R}^{n}, M\right)$. Choosing $\psi\left(z_{n}\right)=$ $z_{n} \frac{3-\epsilon}{4} /\left(1+z_{n}\right)$ yields,

$$
\begin{gathered}
\|F\|_{L^{2}\left(\mathbb{R}^{n}, M\right)}^{2} \leq C \int_{0}^{+\infty} \|\left(\left(t^{2}-1\right) L^{*}\right)^{\frac{2+\epsilon}{4}}\left(\mathrm{I}+\left(t^{2}-\right.\right. \\
\left.1) L^{*}\right)^{-1} F \|_{L^{2}\left(\mathbb{R}^{n}, M\right)}^{2} \frac{d}{d\left(t^{2}-1\right)}
\end{gathered}
$$

Whenever $F \in L_{0}^{2}\left(\mathbb{R}^{n}, M\right)$.

Let $F \in L^{2}\left(\mathbb{R}^{n}, M\right)$. Since

$$
\int_{\mathbb{R}^{n}} L^{*} f\left(x_{n}\right) M\left(x_{n}\right) d x_{n}=0,
$$


it follows from (9) that the same is true with $L^{*(2-\epsilon) / 4} f$. Applying now (12) with $F=L^{*(2-\epsilon) / 4} f$ gives the conclusion of Lemma (3.1).

Let us draw a simple corollary of Lemma (3.1) (see [12]).

Corollary (3.2): For any $\in, \varepsilon>0$, there is $A=A(M, \varepsilon)$ such that

$$
\begin{gathered}
\left\|L^{*(2-\epsilon) / 4} f\right\|_{L^{2}\left(\mathbb{R}^{n}, M\right)}^{2} \leq \tilde{C}_{3} \int_{0}^{A}\left(t^{2}-1\right)^{-\frac{\epsilon-4}{2}} \|\left(t^{2}-\right. \\
1) L^{*}\left(I+\left(t^{2}-1\right) L^{*}\right)^{-1} f \|_{L^{2}\left(\mathbb{R}^{n}, M\right)}^{2}
\end{gathered}
$$

Proof. The proof is straightforward since

$$
\left\|\left(t^{2}-1\right) L^{*}\left(\mathrm{I}+\left(t^{2}-1\right) L^{*}\right)^{-1} f\right\|_{L^{2}\left(\mathbb{R}^{n}, M\right)}^{2} \leq C\|F\|_{L^{2}\left(\mathbb{R}^{n}, M\right)}^{2}
$$

And

$$
\int_{A}^{+\infty}\left(t^{2}-1\right)^{\frac{-3-\epsilon}{2}} d t \overrightarrow{A \rightarrow+\infty} 0
$$

The desired estimate are finally achieved.

Lemma (3.3): Let $\in, \varepsilon>0$ and $A$ given by Corollary (3.2). There exist $\tilde{C}_{4}=\tilde{C}_{4}(M, A)>0$ and $c^{\prime}=c^{\prime}(A, M)>0$ such that, for all $f \in \mathcal{D}\left(\mathbb{R}^{n}\right)$,

$$
\begin{aligned}
& \int_{0}^{A}\left(t^{2}-1\right)^{\frac{\epsilon-4}{2}}\left\|\left(t^{2}-1\right) L^{*}\left(\mathrm{I}+\left(t^{2}-1\right) L^{*}\right)^{-1} f\right\|_{L^{2}\left(\mathbb{R}^{n}, M\right)}^{2} d t \\
& \leq \tilde{C}_{4} \iint_{\mathbb{R}^{n} \times \mathbb{R}^{n}} \frac{\left|f\left(x_{n}\right)-f\left(x_{n+1}\right)\right|^{2}}{\left|x_{n}-x_{n+1}\right|^{n+2-\epsilon}} M\left(x_{n}\right) e^{-c^{\prime}\left|x_{n}-x_{n+1}\right|} d x_{n} d x_{n+1} .
\end{aligned}
$$

Proof: Throughout this proof, for all $x_{n} \in \mathbb{R}^{n}$ and all $s>$ 0 , denote by $\mathcal{Q}\left(x_{n}, s\right)$ the closed cube centered at $x_{n}$ with side length $s$. For fixed $\left(t^{2}-1\right) \in(0, A)$, following Lemma (3.1), Let' us_look for an upper bound for $\|\left(t^{2}-1\right) L^{*}(\mathrm{I}+$ $\left.\left(t^{2}-1\right) L^{*}\right)^{-1} f \|_{L^{2}\left(\mathbb{R}^{n}, M\right)}^{2}$ involving first order differences for $f$. Pick up a countable family of points $\left(x_{n}\right)_{j}^{t^{2}-1} \in \mathbb{R}^{n}, j \in \mathbb{N}$, such that the cubes $\mathcal{Q}\left(\left(x_{n}^{t^{2}-1}\right)_{j}, \sqrt{t^{2}-1}\right)$ have pairwise disjoint interiors, and

$$
\mathbb{R}^{n}=\bigcup_{j \in \mathbb{N}} \mathcal{Q}\left(\left(x_{n}^{t^{2}-1}\right)_{j}, \sqrt{t^{2}-1}\right)
$$

By Lemma (B.1) in [12], there exists a constant $\tilde{C}>0$ such that for all $\in>0$ and all $x_{n} \in \mathbb{R}^{n}$, there are at most $\tilde{C}(1+\epsilon)^{n} \quad$ indexes $j$ such that $\left|x_{n}-\left(x_{n}^{t^{2}-1}\right)_{j}\right| \leq(1+\epsilon) \sqrt{t^{2}-1}$.

For fixed $j$, one has

$$
\begin{aligned}
& \left(t^{2}-1\right) L^{*}\left(\mathrm{I}+\left(t^{2}-1\right) L^{*}\right)^{-1} f \\
& \quad=\left(t^{2}-1\right) L^{*}\left(\mathrm{I}+\left(t^{2}-1\right) L^{*}\right)^{-1} \mathrm{~g}^{j, t^{2}-1}
\end{aligned}
$$

Where, for all $x_{n} \in \mathbb{R}^{n}$,

$$
\mathrm{g}^{j,\left(t^{2}-1\right)}\left(x_{n}\right):=f\left(x_{n}\right)-m^{j,\left(t^{2}-1\right)}
$$

And $m^{j, t^{2}-1}$ is defined by:

$$
:=\frac{1}{\left|Q\left(\left(x_{n}^{t^{2}-1}\right)_{j}, 2 \sqrt{t^{2}-1}\right)\right|} \int_{Q\left(\left(x_{n}^{t^{2}-1}\right)_{j}, 2 \sqrt{t^{2}-1}\right)}^{m^{j, t^{2}-1}} f\left(x_{n+1}\right) d x_{n+1} .
$$

Note that, here, the mean value of $f$ is computed with respect to the Lebesgue measure on $\mathbb{R}^{n}$. Since (14) holds and the cubes $\mathcal{Q}\left(\left(x_{n}^{t^{2}-1}\right)_{j}, 2 \sqrt{t^{2}-1}\right)$ have pairwise disjoint interiors, one clearly has:

$$
\begin{gathered}
\left\|\left(t^{2}-1\right) L^{*}\left(\mathrm{I}+\left(t^{2}-1\right) L^{*}\right)^{-1} f\right\|_{L^{2}\left(\mathbb{R}^{n}, M\right)}^{2}=\sum_{j \in \mathbb{N}}\left\|\left(t^{2}-1\right) L^{*}\left(\mathrm{I}+\left(t^{2}-1\right) L^{*}\right)^{-1} f\right\|_{L^{2} Q\left(\left(x_{n}\right)_{j}^{t^{2}-1}, 2 \sqrt{t^{2}-1}\right)}^{2} \\
=\sum_{j \in \mathbb{N}}\left\|\left(t^{2}-1\right) L^{*}\left(\mathrm{I}+\left(t^{2}-1\right) L^{*}\right)^{-1} \mathrm{~g}^{j, t}\right\|_{L^{2} Q\left(\left(x_{n}^{t^{2}-1}\right)_{j}, 2 \sqrt{t^{2}-1}\right)}^{2},
\end{gathered}
$$

The task of estimating remains,

$$
\left\|\left(t^{2}-1\right) L^{*}\left(\mathrm{I}+\left(t^{2}-1\right) L^{*}\right)^{-1} \mathrm{~g}^{j, t^{2}-1}\right\|_{L^{2} Q}^{2}\left(\left(x_{n}^{t^{2}-1}\right)_{j^{\prime}} 2 \sqrt{t^{2}-1}\right) .
$$

To that purpose, set

$$
C_{0}^{j, t^{2}-1}=L^{2} \mathcal{Q}\left(\left(x_{n}^{t^{2}-1}\right)_{j}, 2 \sqrt{t^{2}-1}\right) \text { and } C_{k}^{j, t^{2}-1}=L^{2} \mathcal{Q}\left(\left(x_{n}^{t^{2}-1}\right)_{j}, 2 \sqrt{t^{2}-1}\right) \backslash L^{2} \mathcal{Q}\left(\left(x_{n}^{t^{2}-1}\right)_{j}, 2 \sqrt{t^{2}-1}\right), \quad \forall k \geq 1,
$$

And $\mathrm{g}_{k}^{j, t^{2}-1}:=\mathrm{g}^{j, t^{2}-1} 1_{C_{k}^{j, t^{2}-1}}, k \geq 0$, where, for any subset $A \subset \mathbb{R}^{n}, 1_{A}$ is the usual characteristic function of $A$. Since $\mathrm{g}^{j, t^{2}-1}=\sum_{k \geq 0} \mathrm{~g}_{k}^{j, t^{2}-1}$ one has:

$$
\left\|\left(t^{2}-1\right) L^{*}\left(\mathrm{I}+\left(t^{2}-1\right) L^{*}\right)^{-1} \mathrm{~g}^{j, t^{2}-1}\right\|_{L^{2} Q}^{2}\left(\left(x_{n}^{t^{2}-1}\right)_{j}, 2 \sqrt{t^{2}-1}\right)
$$




$$
\leq \sum_{k \geq 0}\left\|\left(t^{2}-1\right) L^{*}\left(\mathrm{I}+\left(t^{2}-1\right) L^{*}\right)^{-1} \mathrm{~g}^{j, t^{2}-1}\right\|_{L^{2} Q}^{2}\left(\left(x_{n}^{t^{2}-1}\right)_{j^{2}} 2 \sqrt{t^{2}-1}\right)
$$

and, using Lemma (2.1), one obtains (for some constants $\tilde{C}, \tilde{c}>0$ ):

$$
\begin{gathered}
\left\|\left(t^{2}-1\right) L^{*}\left(\mathrm{I}+\left(t^{2}-1\right) L^{*}\right)^{-1} \mathrm{~g}^{j, t^{2}-1}\right\|_{L^{2} Q}^{2}\left(\left(x_{n}^{t^{2}-1}\right)_{j^{2}}{ }^{2} \sqrt{t^{2}-1}\right) \\
\leq \tilde{C}\left(\left\|\mathrm{~g}_{0}^{j, t^{2}-1}\right\|_{L^{2}\left(c_{0}^{j, t^{2}-1}, M\right)}+\sum_{k \geq 0} e^{-\tilde{c} 2^{k}}\left\|\mathrm{~g}_{k}^{j, t^{2}-1}\right\|_{L^{2}\left(C_{k}^{j, t^{2}-1}, M\right)}\right)
\end{gathered}
$$

By Cauchy-Schwarz's inequality, the deduction (for another constant $C^{\prime}{ }_{1}>0$ ):

$$
\begin{gathered}
\left\|\left(t^{2}-1\right) L^{*}\left(\mathrm{I}+\left(t^{2}-1\right) L^{*}\right)^{-1} \mathrm{~g}^{j, t^{2}-1}\right\|_{L^{2} Q}^{2}\left(\left(x_{n}^{t^{2}-1}\right)_{j^{\prime}}, 2 \sqrt{t^{2}-1}\right) \\
\leq C^{\prime}{ }_{1}\left(\left\|\mathrm{~g}_{0}^{j, t^{2}-1}\right\|_{L^{2}\left(C_{0}^{j, t^{2}-1}, M\right)}+\sum_{k \geq 0} e^{-\tilde{c} 2^{k}}\left\|\mathrm{~g}_{k}^{j, t^{2}-1}\right\|_{L^{2}\left(c_{k}^{j, t^{2}-1}, M\right)}\right)
\end{gathered}
$$

As a consequence:

$$
\begin{aligned}
& \int_{0}^{A}\left(t^{2}-1\right)^{\frac{\epsilon-4}{2}}\left\|\left(t^{2}-1\right) L^{*}\left(\mathrm{I}+\left(t^{2}-1\right) L^{*}\right)^{-1} \mathrm{~g}^{j, t^{2}-1}\right\|_{L^{2} Q\left(\left(x_{n}^{t^{2}-1}\right)_{j^{\prime}}, 2 \sqrt{t^{2}-1}\right)}^{2} d\left(t^{2}-1\right) \\
& \left.\leq C^{\prime} \int_{0}^{A}\left(t^{2}-1\right)^{\frac{-(3-\epsilon)}{2}} \sum_{j \geq 0}\left\|\mathrm{~g}_{0}^{j, t^{2}-1}\right\|_{L^{2}\left(c_{0}^{j, t^{2}-1}, M\right)} d\left(t^{2}-1\right)\right) \\
& +C^{\prime}{ }_{1} \int_{0}^{A}\left(t^{2}-1\right)^{\frac{-(3-\epsilon)}{2}} \sum_{k \geq 1} e^{-\tilde{c} 2^{k}} \sum_{j \geq 0}\left\|\mathrm{~g}_{k}^{j, t^{2}-1}\right\|_{L^{2}\left(c_{k}^{j, t^{2}-1}, M\right)} d\left(t^{2}-1\right) .
\end{aligned}
$$

It can be claimed that

Lemma (3.4): There exists $\overline{\mathrm{C}}_{1}>0$ such that, for all $\mathrm{t}^{2}>1$ and all $\mathrm{j} \in \mathbb{N}$ :

A. For the first term:

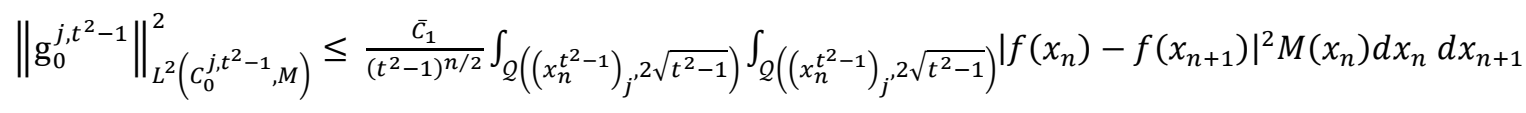

B. For all $k \geq 1$,

$$
\left\|\mathrm{g}_{k}^{j, t^{2}-1}\right\|_{L^{2}\left(c_{k}^{j, t^{2}-1, M}\right)}^{2} \leq \frac{\bar{C}_{1}}{\left(\sqrt{t^{2}-1}\right)^{2}} \int_{x_{n} \in Q\left(\left(x_{n}^{t^{2}-1}\right)_{j^{\prime}} 2^{k+1} \sqrt{t^{2}-1}\right)} \int_{y_{n+1} \in Q\left(\left(x_{n}^{t^{2}-1}\right)_{j^{2}},^{k+1} \sqrt{t^{2}-1}\right)}\left|f\left(x_{n}\right)-f\left(x_{n+1}\right)\right|^{2} M\left(x_{n}\right) d x_{n} d x_{n+1}
$$

The proof shall be postponed to the end of the section followed by the proof of Lemma (3.3). Using Assertion A in Lemma (3.4), summing up on $j \geq 0$ and integrating over $(0, A)$, the result

$$
\begin{aligned}
& \int_{0}^{A}\left(t^{2}-1\right)^{\frac{-(3-\epsilon)}{2}} \sum_{j \geq 0}\left\|\mathrm{~g}_{0}^{j, t^{2}-1}\right\|_{L^{2}\left(c_{0}^{j, t^{2}-1}, M\right)}^{2} d\left(t^{2}-1\right)=\sum_{j \geq 0} \int_{0}^{A}\left(t^{2}-1\right)^{\frac{-(3-\epsilon)}{2}}\left\|\mathrm{~g}_{0}^{j, t^{2}-1}\right\|_{L^{2}\left(c_{0}^{j, t^{2}-1}, M\right)}^{2} d\left(t^{2}-1\right) \\
\leq & \bar{C}_{1} \sum_{j \geq 0} \int_{0}^{A}\left(t^{2}-1\right)^{\frac{-(3+\epsilon+n)}{2}}\left(\int_{Q\left(\left(x_{n}\right)_{j}^{\left.t^{2}-1,2 \sqrt{t^{2}-1}\right)}\right.} \int_{Q\left(\left(x_{n}^{t^{2}-1}\right)_{j^{2}} 2 \sqrt{t^{2}-1}\right)}\left|f\left(x_{n}\right)-f\left(x_{n+1}\right)\right|^{2} M\left(x_{n}\right) d x_{n} d x_{n+1}\right) d\left(t^{2}-1\right)
\end{aligned}
$$




$$
\leq \bar{C}_{1} \sum_{j \geq 0} \iint_{\left(x_{n}, x_{n+1}\right) \in \mathbb{R}^{n} \times \mathbb{R}^{n}}\left|f\left(x_{n}\right)-f\left(x_{n+1}\right)\right|^{2} M\left(x_{n}\right)\left(\int_{t \geq \max \left\{\frac{\left|x_{n}-\left(x_{n}^{2}-1\right) j_{j}\right|^{2}}{n} ; \frac{x_{n+1}-\left.\left(x_{n}^{t_{n}^{2}-1}\right)_{j}\right|^{2}}{n}\right\}}^{A}\left(t^{2}-1\right)^{\frac{-(3+\epsilon+n)}{2}} d\left(t^{2}-1\right)\right) d x_{n} d x_{n+1}
$$

The Fubini theorem now shows:

$$
\begin{aligned}
& \sum_{j \geq 0} \quad \int_{\left.\left(t^{2}-1\right)\right|^{2} \mid t_{n+1-\left.\left(t^{2}-1\right)\right|^{2}}}^{A}\left(t^{2}-1\right)^{\frac{-(3+\epsilon+n)}{2}} d\left(t^{2}-1\right) \\
& \left(t^{2}-1\right) \geq \max \left\{\frac{\left|x_{n}-\left(x_{n}^{t^{2}-1}\right)_{j}\right|^{2}}{n}: \frac{\mid x_{n+1}-\left(x_{n}^{t^{2}-1}\right)_{j}}{n}\right\}
\end{aligned}
$$

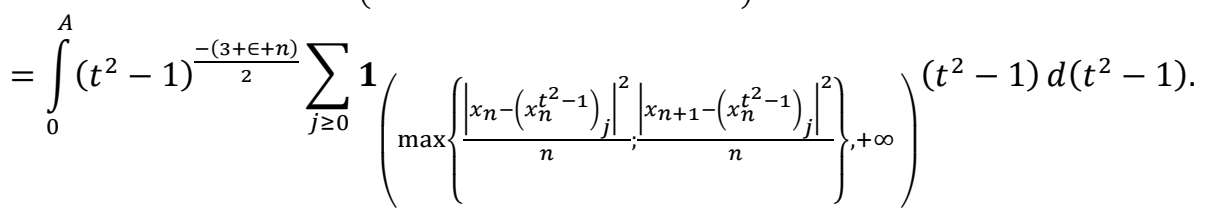

Observe that, by Lemma (B.1) in [12], there is a constant $N \in \mathbb{N}$ such that, for all $t^{2}>1$, there are at most $N$ indexes $j$ such that $\left|x_{n}-\left(x_{n}^{t^{2}-1}\right)_{j}\right|^{2}<n\left(t^{2}-1\right)$ and $\left|x_{n+1}-\left(x_{n}^{t^{2}-1}\right)_{j}\right|^{2}<n\left(t^{2}-1\right)$. If such an index $j$ exists, one has $\left|x_{n}-x_{n+1}\right|<$ $2 \sqrt{n\left(t^{2}-1\right)}$. It therefore follows that

$$
\sum_{j \geq 0} \mathbf{1}\left(\max \left\{\frac{\left|x_{n}-\left(x_{n}^{t^{2}-1}\right)_{j}\right|^{2}}{n} ; \frac{\left|x_{n+1}-\left(x_{n}^{t^{2}-1}\right)_{j}\right|^{2}}{n}\right\},+\infty\right)^{\left(t^{2}-1\right) \leq N \mathbf{1}_{\left(\left|x_{n}-x_{n+1}\right|^{2} / 4 n,+\infty\right)}\left(t^{2}-1\right)}
$$

So that

$$
\begin{gathered}
\int_{0}^{A}\left(t^{2}-1\right)^{\frac{\epsilon-4}{2}} \sum_{j}\left\|\mathrm{~g}_{0}^{j, t^{2}-1}\right\|_{L^{2}\left(c_{0}^{j, t^{2}-1}, M\right)}^{2} d t \\
\leq \bar{C} N \int_{\mathbb{R}^{n} \times \mathbb{R}^{n}}\left|f\left(x_{n}\right)-f\left(x_{n+1}\right)\right|^{2} M\left(x_{n}\right)\left(\int_{\left|x_{n}-x_{n+1}\right|^{2} / 4 n}^{A}\left(t^{2}-1\right)^{\frac{-(4+\epsilon+n)}{2}} d\left(t^{2}-1\right)\right) d x_{n} d x_{n+1} \\
\leq \bar{C}_{1} N \iint_{\left|x_{n}-x_{n+1}\right| \leq 2 \sqrt{n A}} \frac{\left|f\left(x_{n}\right)-f\left(x_{n+1}\right)\right|^{2}}{\left|x_{n}-x_{n+1}\right|^{n+2-\epsilon}} M\left(x_{n}\right) d x_{n} d x_{n+1}
\end{gathered}
$$

Using Assertion B in Lemma (3.4), it is obtained that, for all $j \geq 0$ and all $k \geq 1$,

$$
\begin{gathered}
\int_{0}^{A}\left(t^{2}-1\right)^{\frac{\epsilon-4}{2}} \sum_{j \geq 0}\left\|\mathrm{~g}_{k}^{j, t^{2}-1}\right\|_{2}^{2} d\left(t^{2}-1\right) \\
\leq \bar{C}_{1} \sum_{j \geq 0} \int_{0}^{A}\left(t^{2}-1\right)^{-1-(2-\epsilon) / 2}\left(\int_{Q\left(\left(x_{n}^{t^{2}-1}\right)_{j^{2}}{ }^{k+1} \sqrt{t^{2}-1}\right) \times Q} \int_{\left(\left(x_{n}^{t^{2}-1}\right)_{j^{2}}{ }^{k+1} \sqrt{t^{2}-1}\right)}\left|f\left(x_{n}\right)-f\left(x_{n+1}\right)\right|^{2} M\left(x_{n}\right) d x_{n} d x_{n+1}\right) d\left(t^{2}-1\right)
\end{gathered}
$$




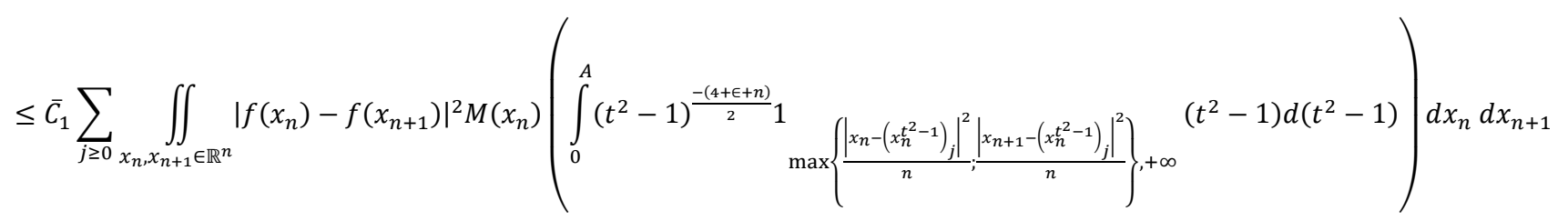

But, given $\mathrm{t}^{2}>1, x_{n}, x_{n+1} \in \mathbb{R}^{\mathrm{n}}$, by Lemma (B.1) in [12] again, there exist at most $\tilde{\mathrm{C}}_{1} 2^{\mathrm{kn}}$ indexes $\mathrm{j}$ such that

$$
\left|x_{n}-\left(x_{n}^{t^{2}-1}\right)_{j}\right| \leq 2^{k} \sqrt{n\left(t^{2}-1\right)} \text { and }\left|x_{n+1}-\left(x_{n}^{t^{2}-1}\right)_{j}\right| \leq 2^{k} \sqrt{n\left(t^{2}-1\right)},
$$

and for these indexes $j,\left|x_{n}-x_{n+1}\right| \leq 2^{k+1} \sqrt{n\left(t^{2}-1\right)}$. As a consequence we have:

$$
\begin{aligned}
& \int_{0}^{A}\left(t^{2}-1\right)^{\frac{-(4+\epsilon+n)}{2}} \sum_{j \geq 0} \mathbf{1} \max \left\{\frac{\left|x_{n}-\left(x_{n}^{t^{2}-1}\right) j_{j}\right|}{4^{k} n} ; \frac{\left|x_{n+1}-\left(x_{n}^{t^{2}-1}\right)_{j}\right|^{2}}{4^{k} n}\right\},+\infty \\
\leq & \tilde{C}_{1} 2^{k n} \int_{t^{2} \geq \frac{\left|x_{n}-x_{n+1}\right|^{2}}{4^{k+1} n}+1}^{A}\left(t^{2}-1\right)^{-\frac{-(4+\epsilon+n)}{2}} d t \leq \tilde{C}_{1}^{\prime 2 k(2-\epsilon+n)}\left|x_{n}-x_{n+1}\right|^{-n-(2-\epsilon)} \mathbf{1}_{\left|x_{n}-x_{n+1}\right| \leq 2^{k+1 \sqrt{n A}},}
\end{aligned}
$$

for some other constant $\tilde{C}^{\prime}{ }_{1}>0$, and therefore

$\int_{0}^{A}\left(t^{2}-1\right)^{-1-\frac{2-\epsilon}{2}} \sum_{j}\left\|\mathrm{~g}_{k}^{j, t^{2}-1}\right\|_{L^{2}\left(C_{0}^{j, t^{2}-1}, M\right)}^{2} d t \leq \bar{C}_{1} \tilde{C}^{\prime}{ }^{\prime} 2^{k(2-\epsilon+n)} \int_{\left|x_{n}-x_{n+1}\right| \leq 2^{k+1} \sqrt{n A}} \frac{\left|f\left(x_{n}\right)-f\left(x_{n+1}\right)\right|^{2}}{\left|x_{n}-x_{n+1}\right|^{n+2-\epsilon}} M\left(x_{n}\right) d x_{n} d x_{n+1}$.

Concluding the proof of Lemma (3.3), using Lemma (3.1), (16), (19) and (20). It is thus proved, by reconsidering (18):

$$
\begin{gathered}
\int_{0}^{A}\left(t^{2}-1\right)^{\frac{(\epsilon-4)}{2}}\left\|\left(t^{2}-1\right) L^{*}\left(\mathrm{I}+\left(t^{2}-1\right) L^{*}\right)^{-1} f\right\|_{L^{2}\left(\mathbb{R}^{n}, M\right)}^{2} d\left(t^{2}-1\right) \\
\leq C^{\prime}{ }_{1} \tilde{C}_{1} N \int_{\left|x_{n}-x_{n+1}\right| \leq 2^{k+1} \sqrt{n A}} \frac{\left|f\left(x_{n}\right)-f\left(x_{n+1}\right)\right|^{2}}{\left|x_{n}-x_{n+1}\right|^{n+2-\epsilon}} M\left(x_{n}\right) d x_{n} d x_{n+1} \\
\quad+\sum_{k \geq 1} C^{\prime}{ }_{1} \bar{C}_{1} \tilde{C}^{\prime}{ }_{1} 2^{k(2-\epsilon)} e^{-\tilde{c} 2^{k}} \iint_{\left|x_{n}-x_{n+1}\right| \leq 2^{k+1} \sqrt{n A}} \frac{\left|f\left(x_{n}\right)-f\left(x_{n+1}\right)\right|^{2}}{\left|x_{n}-x_{n+1}\right|^{n+2-\epsilon}} M\left(x_{n}\right) d x_{n} d x_{n+1}
\end{gathered}
$$

Hence, the deduction:

$$
\begin{aligned}
& \int_{0}^{A}\left(t^{2}-1\right)^{\frac{(\epsilon-4)}{2}}\left\|\left(t^{2}-1\right) L^{*}\left(\mathrm{I}+\left(t^{2}-1\right) L^{*}\right)^{-1} f\right\|_{L^{2}\left(\mathbb{R}^{n}, M\right)}^{2} d\left(t^{2}-1\right) \\
& \leq \tilde{C}_{4} \iint_{\mathbb{R}^{n} \times \mathbb{R}^{n}} \frac{\left|f\left(x_{n}\right)-f\left(x_{n+1}\right)\right|^{2}}{\left|x_{n}-x_{n+1}\right|^{n+2-\epsilon}} M\left(x_{n}\right) e^{-c^{\prime}\left|x_{n}-x_{n+1}\right|} d x_{n} d x_{n+1}
\end{aligned}
$$

for some constants $\tilde{C}_{4}$ and $c^{\prime}>0$ as claimed in the statement.

Proof of Lemma (3.4): Observe first that, for all $\mathrm{x}_{\mathrm{n}} \in \mathbb{R}^{\mathrm{n}}$,

$$
\begin{gathered}
\mathrm{g}_{0}^{j, t^{2}-1}\left(x_{n}\right)=f\left(x_{n}\right)-\frac{1}{\left|\mathcal{Q}\left(\left(x_{n}^{t^{2}-1}\right)_{j}, 2 \sqrt{t^{2}-1}\right)\right|} \int_{Q\left(x_{j}^{t^{2}-1}, 2 \sqrt{t^{2}-1}\right)} f\left(x_{n+1}\right) d x_{n+1} . \\
=\frac{1}{\left|\mathcal{Q}\left(\left(x_{n}^{t^{2}-1}\right)_{j^{\prime}}, 2 \sqrt{t^{2}-1}\right)\right|_{Q\left(\left(x_{n}^{t^{2}-1}\right)_{j^{\prime}}, 2 \sqrt{t^{2}-1}\right)}\left(f\left(x_{n}\right)-f\left(x_{n+1}\right)\right) d x_{n+1} .}
\end{gathered}
$$

By Cauchy-Schwarz inequality, it follows that 


$$
\left|\mathrm{g}_{0}^{j, t^{2}-1}\left(x_{n}\right)\right|^{2} \leq \frac{\tilde{C}}{\left(t^{2}-1\right)^{n / 2}} \underset{Q\left(\left(x_{n}^{t^{2}-1}\right)_{j^{2}}{ }^{2} \sqrt{t^{2}-1}\right)}{ }\left|f\left(x_{n}\right)-f\left(x_{n+1}\right)\right|^{2} d x_{n+1}
$$

Therefore,

$$
\left\|\mathrm{g}_{k}^{j, t^{2}-1}\right\|_{L^{2}\left(c_{k}^{j, t}, M\right)}^{2} \leq \frac{\tilde{C}}{\left(t^{2}-1\right)^{n / 2}} \int_{Q\left(\left(x_{n}^{t^{2}-1}\right)_{j^{\prime}} 2 \sqrt{t^{2}-1}\right)} \int_{\left(\left(x_{n}^{t^{2}-1}\right)_{j^{2}}{ }^{2} \sqrt{t^{2}-1}\right)}\left|f\left(x_{n}\right)-f\left(x_{n+1}\right)\right|^{2} M\left(x_{n}\right) d x_{n} d x_{n+1}
$$

Which shows Assertion A. Similarly, the argument for Assertion B and obtain:

$$
\left\|\mathrm{g}_{k}^{j, t^{2}-1}\right\|_{L^{2}\left(c_{k}^{j, t^{2}-1}, M\right)}^{2} \leq \frac{\tilde{C}}{t^{n / 2}} \int_{x \in Q} \int_{\left(\left(x_{n}^{t^{2}-1}\right)_{j^{\prime}} 2 \sqrt{t^{2}-1}\right)} \int_{y \in Q\left(\left(x_{n}^{t^{2}-1}\right)_{j^{\prime}} 2 \sqrt{t^{2}-1}\right)}\left|f\left(x_{n}\right)-f\left(x_{n+1}\right)\right|^{2} M\left(x_{n}\right) d x_{n} d x_{n+1},
$$

which ends the proof of Lemma (3.4)

A few concluding remarks on Lemma (3.4). It is a well-known fact [33] that, when $\epsilon \geq 0$.

$$
\left\|(-\Delta)^{(2-\epsilon) / 4} f\right\|_{L^{1+\epsilon,}\left(\mathbb{R}^{n}\right)} \leq \tilde{C}_{2-\epsilon, 1+\epsilon,}\left\|S_{2-\epsilon, 1+\epsilon, f}\right\|_{L^{1+\epsilon,\left(\mathbb{R}^{n}\right)}}
$$

Where

$$
S_{2-\epsilon, 1+\epsilon} f\left(x_{n}\right)=\left(\int_{0}^{+\infty}\left(\int_{B}\left|f\left(x_{n}+r x_{n+1}\right)-f\left(x_{n}\right)\right| d x_{n+1}\right)^{2} \frac{d r}{r^{3-\epsilon}}\right)^{\frac{1}{2}}
$$

And also [35]

$$
\left\|(-\Delta)^{\frac{2-\epsilon}{4}} f\right\|_{L^{1+\epsilon}\left(\mathbb{R}^{n}\right)} \leq \tilde{C}_{2-\epsilon, 1+\epsilon}\left\|D_{2-\epsilon} f\right\|_{L^{1+\epsilon}\left(\mathbb{R}^{n}\right)}
$$

Where

$$
D_{2-\epsilon} f\left(x_{n}\right)=\left(\int_{\mathbb{R}^{n}} \frac{\left|f\left(x_{n}+x_{n+1}\right)-f\left(x_{n}\right)\right|^{2}}{\left|x_{n+1}\right|^{n+2-\epsilon}} d x_{n+1}\right)^{\frac{1}{2}}
$$

In [35], these inequalities were extended to the setting of a unimodular Lie group endowed with a sub-laplacian $\Delta$, relying on semigroups techniques and Littlewood-PaleyStein functionals. In particular, in [35] and [12] use pointwise estimates of the kernel of the semigroup generated by $\Delta$. The conclusion of Lemma (3.4) means that the norm of $L^{*(2-\epsilon) / 4} f$ in $L^{2}\left(\mathbb{R}^{n}, M\right)$ is bounded from above by the $L^{2}\left(\mathbb{R}^{n}, M\right)$ norm of an appropriate version of $D_{2-\epsilon}$. Note that this does not require pointwise estimates for the kernel of the semigroup generated by $L^{*}$, and that the $L^{2}$ off-diagonal estimates given by Lemma (2.1), which hold for a general sequence measure $M$, are enough for the argument to hold (see [12]) However, it remains uncertain_if an $L^{1+\epsilon}$ version of Lemma (3.4) still holds. Note also that we do not compare the $L^{2}\left(\mathbb{R}^{n}, M\right)$ norm of $L^{*(2-\epsilon) / 4} f$ with the $L^{2}\left(\mathbb{R}^{n}, M\right)$ norm of a version of $S_{2-\epsilon, 1+\epsilon} f$. Finally, the converse inequalities to (22) and (23) hold in $\mathbb{R}^{n}$ and also on a unimodular Lie group [36] and [12] did not consider the corresponding inequalities.

\section{4. $\left\|L^{*(2-\epsilon) / 4} f\right\|_{L^{2}\left(\mathbb{R}^{n}, M\right)}$ for theControl of the Moment of $\boldsymbol{f}$ and Proof of Theorem (1.2)}

Observe first that, by the definition of $L^{*}$, the finding

$$
\int_{\mathbb{R}^{n}}\left|\nabla f\left(x_{n}\right)\right|^{2} M\left(x_{n}\right) d x_{n}=\int_{\mathbb{R}^{n}} L^{*} f\left(x_{n}\right) f\left(x_{n}\right) M\left(x_{n}\right) d x_{n},
$$

for all $f \in \mathcal{D}\left(L^{*}\right)$. The inequality (4) can therefore be rewritten, in terms of operators, as

$$
L^{*} \geq \lambda^{\prime} \mu
$$

Where $\mu$ is the multiplication operator by $x_{n} \mapsto 1+$ $\left|\nabla \ln M\left(x_{n}\right)\right|^{2}$. Since $\mu$ is a nonnegative operator on $L^{2}\left(\mathbb{R}^{n}, M\right)$, using a functional calculus argument (see [36]), one deduces from (24) that, for any $\in<2$, 


$$
L^{* \frac{2-\epsilon}{2}} \geq\left(\lambda^{\prime}\right)^{\frac{2-\epsilon}{2}}(\mu)^{\frac{2-\epsilon}{2}},
$$

Which implies, thanks to the fact $L^{* \frac{2-\epsilon}{2}}=\left(L^{* \frac{2-\epsilon}{4}}\right)^{2}$ and the symmetry of $L^{* \frac{2-\epsilon}{4}}$ on $L^{2}\left(\mathbb{R}^{n}, M\right)$, that

$$
\begin{aligned}
& \left(\lambda^{\prime}\right)^{(2-\epsilon) / 2} \int_{\mathbb{R}^{n}}\left|f\left(x_{n}\right)\right|^{2}\left(1+\left|\nabla \ln M\left(x_{n}\right)\right|^{2}\right)^{\frac{2-\epsilon}{2}} M\left(x_{n}\right) d x_{n} \\
& \leq \int_{\mathbb{R}^{n}}\left|L^{* \frac{2-\epsilon}{4}} f\left(x_{n}\right)\right|^{2} M\left(x_{n}\right) d x_{n} \\
& =\left\|L^{* \frac{2-\epsilon}{4}} f\right\|_{L^{2}\left(\mathbb{R}^{n}, M\right)}^{2} \text {. }
\end{aligned}
$$

The conclusion of Theorem (1.2) readily follows using the previous inequality in conjunction with Corollary (3.2) and Lemma (3.3), and picking $\varepsilon$ small enough.

Corollary (4.1): If $L^{*}$ is self-adjoint and normal then

(i) $\left\|\lambda^{\prime}\right\|_{L^{2}} \geq \frac{\operatorname{dist}\left(\mu, \Sigma L^{*}\right)}{\|\mu\|_{L^{2}}}-\epsilon$.

(ii) $\|\mu\|_{L^{2}} \leq \frac{1}{\left|\lambda^{\prime}\right|}$.

(iii) I $>2-\mathrm{t}^{2}$.

(iv) $\left\|\mathrm{L}^{*}\right\|_{\mathrm{L}^{2}}<1+\frac{\epsilon}{\mathrm{t}^{2}-1}$.

Proof:

(i) Since $L^{*} \geq \lambda^{\prime} \mu$ then $\left\|\left(L^{*}-\mu\right)^{-1}\right\|_{L^{2}} \leq \| \mu^{-1}\left(\lambda^{\prime}-\right.$ 1) $)^{-1} \|_{L^{2}}$

(ii) The result t $\left\|\mu\left(\lambda^{\prime}-1\right)\right\|_{L^{2}}=\operatorname{dist}\left(\mu, \sum L^{*}\right)-\epsilon$

(iii) Thus, $\left\|\lambda^{\prime}\right\|_{L^{2}} \geq \frac{\operatorname{dist}\left(\mu, \Sigma L^{*}\right)}{\|\mu\|_{L^{2}}}-\epsilon$.

(iv) Let $L^{*}$ be a contraction from (24) leads to $\|\mu\|_{L^{2}} \leq \frac{1}{\left|\lambda^{\prime}\right|}$

(v) Given $\left\|\left(I+\left(\mathrm{t}^{2}-1\right) \lambda^{\prime} \mu\right)^{-1}\right\| \leq 1$, using (iiresulting in $\mathrm{I}>2-\mathrm{t}^{2}$

(vi) For $\left\|\left(I+\left(\mathrm{t}^{2}-1\right) L^{*}\right)^{-1}\right\| \leq 1$, and $I+\left(\mathrm{t}^{2}-1\right) L^{*}=$ $1+\epsilon$, using (iii) to conclude that $\left\|\mathrm{L}^{*}\right\|_{\mathrm{L}^{2}}<1+\frac{\epsilon}{\mathrm{t}^{2}-1}$

\section{References}

[1] D. del Castillo-Negrete, B. Carreras, V. Lynch, Nondiffusive transport in plasma turbulence: a fractional diffusion approach, Phys. Rev. Lett.94 (2005) 065003.

[2] L. Caffarelli, A. Vasseur, Drift diffusion equations with fractional diffusion and the quasi-geostrophic equation, Ann. of Math. 171 (3) (2010)1903-1930.

[3] C. Villani, On a new class of weak solutions to the spatially homogeneous Boltzmann and Landau equations, Arch. Ration. Mech. Anal. 143(1998) 273-307.
[4] C. Mouhot, Explicit coercivity estimates for the linearized Boltzmann and Landau operators, Comm. Partial Differential Equations 31 (2006)1321-1348.

[5] C. Mouhot, R.M. Strain, Spectral gap and coercivity estimates for linearized Boltzmann collision operators without angular cutoff, J. Math.Pures Appl. (9) 87 (2007) 515-535.

[6] P. Gressman, R. Strain, Global classical solutions of the Boltzmann equation with long-range interactions, Proc. Natl. Acad. Sci. USA 107(2010) 5744-5749.

[7] G. Di Nunno, B. Øksendal, F. Proske, Malliavin Calculus for Lévy Processes with Applications to Finance, Universitext, Springer-Verlag,Berlin, 2009.

[8] W. Feller, An Introduction to Probability Theory and Its Applications. Vol. II, second edition, John Wiley \& Sons Inc., New York, 1971.

[9] A. Signorini, Questioni di elasticità non linearizzata e semilinearizzata, Rend. Mat. Appl. (5) 18 (1959) 95-139.

[10] L. Silvestre, Regularity of the obstacle problem for a fractional power of the Laplace operator, Comm. Pure Appl. Math. 60 (2007) 67-112.

[11] L. Caffarelli, A. Figalli, Regularity of solutions to the parabolic fractional obstacle problem, preprint, 2010.

[12] C. Mouhot, E. Russ and Y. Sire. Fractional Poincaré inequalities for general measures J. Math. Pures Appl. 95 (2011) 72-84.

[13] R. Adams, J. Fourier, Sobolev Spaces, second edition, Pure Appl. Math.(Amst.), vol. 140, Elsevier/Academic Press, Amsterdam, 2003.

[14] D. Bakry, F. Barthe, P. Cattiaux, A. Guillin, A simple proof of the Poincaré inequality for a large class of probability measures including thelog-concave case, Electron. Commun. Probab. 13 (2008) 60-66.

[15] M. Kanai, Rough isometries and combinatorial approximations of geometries of noncompact Riemannian manifolds, J. Math. Soc. Japan 37(1985) 391-413.

[16] X. Chen, Feng-Y. Wang and J. Wang, Perturbations of functional inequalities for Lévy type Dirichlet forms, Forum Mathematicum (June 2014). Volume 27, Issue 6, ISSN 0933-7741, Pages 3477 3507 .

[17] X. Chen, J.Wang Intrinsic ultracontractivity of Feynman-Kac semigroups for symmetric jump processes, Journal of Functional Analysis(1 June 2016), Volume 270, Issue 11, Pages 4152-4195.

[18] Wang J. A simple approach to functional inequalities for nonlocal Dirichlet forms. ESAIM Probab Stat, 2014, 18: 503-513.

[19] Chen X, Wang J. Functional inequalities for nonlocal Dirichlet forms with finite range jumps or large jumps. Stochastic Process Appl, 2014, 124: 123-153.

[20] C. Villani, Hypocoercivity, Mem. Amer. Math. Soc. 202 (2009) $1-141$.

[21] J.-D. Deuschel, D. W. Stroock, Hypercontractivity and spectral gap of symmetric diffusions with applications to the stochastic Ising models,J. Funct. Anal. 92 (1990) 30-48.

[22] M. Ledoux, The Concentration of Measure Phenomenon, Amer. Math. Soc., 2001. 
[23] L. Wu, A new modified logarithmic Sobolev inequality for Poisson point processes and several applications, Probab. Theory Related Fields 118(2000) 427-438.

[24] D. Chafaï, Entropies, convexity, and functional inequalities: on $\Phi$-entropies and $\Phi$-Sobolev inequalities, J. Math. Kyoto Univ. 44 (2004)325-363.

[25] I. Gentil, C. Imbert, The Lévy-Fokker-Planck equation:Фentropies and convergence to equilibrium, Asymptot. Anal. 59 (2008) 125-138.

[26] N. S. Landkof, Foundations of Modern Potential Theory, Grundlehren Math.Wiss., Band 180, Springer-Verlag, New York, 1972, translatedfrom the Russian by A.P. Doohovskoy.

[27] D. Bakry, M. Émery, Propaganda for $\Gamma 2$, in: From Local Times to Global Geometry, Control and Physics, Coventry, 1984/85, in: Pitman Res.Notes Math. Ser., vol. 150, Longman Sci. Tech., Harlow, 1986, pp. 39-46.

[28] M. Gaffney, The conservation property of the heat equation on Riemannian manifolds, Comm. Pure Appl. Math. 12 (1959) 1-11.

[29] P. Auscher, On Necessary and Sufficient Conditions for $L_{p}$ Estimates of Riesz Transforms Associated to Elliptic
Operators on $\mathrm{R}^{\mathrm{n}}$ and Related Estimates, Mem. Amer. Math. Soc., vol. 186, Amer. Math.Soc., 2007.

[30] P. Auscher, S. Hofmann, M. Lacey, A. McIntosh, P. Tchamitchian, The solution of the Kato square root problem for second order ellipticoperators onR ${ }^{n}$, Ann. of Math. (2) 156 (2002) 633-654.

[31] P. Auscher, A. McIntosh, E. Russ, Hardy spaces of differential forms on Riemannian manifolds, J. Geom. Anal. 18 (2008) 192-248.

[32] D. Henry, Geometric Theory of Semilinear Parabolic Equations, second edition, Lecture Notes in Math., vol. 840, Springer-Verlag, Berlin/New York, 1981.

[33] R. Strichartz, Multipliers on fractional Sobolev spaces, J. Math. Mech. 16 (1967) 1031-1060.

[34] E. Stein, The characterization of functions arising as potentials I, Bull. Amer. Math. Soc. 67 (1961) 102-104.

[35] T. Coulhon, E. Russ, V. Tardivel-Nachef, Sobolev algebras on Lie groups and Riemannian manifolds, Amer. J. Math. 123 (2001) 283-342.

[36] E. Davies, One-Parameter Semigroups, second edition, London Math. Soc. Monogr. Ser., vol. 15, Academic Press Inc., London/New York, 1980. 